\title{
Die SÄZ ist auf Kurs!
}

\section{Bruno Kesseli}

Dr. med. et lic. phil., Chefredaktor

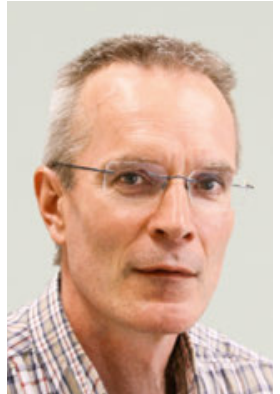

20 Jahre jung ist der Schweizerische Ärzteverlag EMH im zu Ende gehenden Jahr geworden, hat seine jugendliche «Sturm-und-Drang»-Phase damit gewissermassen beendet. Doch in ruhigen Gewässern segelt die EMH-Flotte deswegen noch lange nicht.

Die etwas forcierte Metaphorik ist bewusst gewählt, denn bildliche Umschreibungen der allgemeinen Lage hatten rund um das Jubiläum Hochkonjunktur. Verwaltungsratspräsident Hans Kurt, dessen Jubiläumsartikel in diesem Heft zu lesen ist [1], sprach am Treffen von EMH mit den Delegierten der Fachgesellschaften von "dunklen Wolken und blauem Himmel» [2]. Damit zielte er einerseits auf die anstehenden Herausforderungen in Bezug auf die Finanzierung unabhängiger, qualitativ hochwertiger Fachzeitschriften, andererseits auf die Chance, die aktuelle Dynamik der Medienwelt mit einer intelligenten Verlagsstrategie zur Stärkung des eigenen Unternehmens zu nutzen.

Was die Schweizerische Ärztezeitung (SÄZ) im Speziellen betrifft, hatte der Schreibende an dieser Stelle schon vor einem Jahr eine meteorologische Metapher bemüht. "Die SÄZ will rauen Winden trotzen", lautete damals vor dem Hintergrund der zunehmend schwierigen ökonomischen Rahmenbedingungen die Ansage. Diese Botschaft gilt nach wie vor. Doch ist das «Flaggschiff», als das die SÄZ gelegentlich bezeichnet wird, überhaupt auf Kurs? Bringt es seine Fracht ans Ziel, erfüllt es seine Funktion als offizielles Organ der FMH und freie Diskussionsplattform für die Ärzteschaft und weitere am Gesundheitswesen beteiligte Kreise? Seit diesem Jahr können wir solche Fragen verlässlicher beantworten als früher. Denn die im Sommer durchgeführte «Leserumfrage Medizinische Fachpresse 2017» des renommierten Markt- und Sozialforschungsinstituts gfs-zürich liefert im Gegensatz zur ersten, 2010 durchgeführten Befragung auch detaillierte qualitative Daten zu den 48 untersuchten Zeitschriften [3]. Die Grösse der Stichprobe mit 2550 teilnehmenden Ärztinnen und Ärzten lässt zu einer Vielzahl von spezifischen Fragestellungen valide Aussagen zu. Es ist nicht vermessen zu behaupten, dass sich die Ergebnisse der SÄZ sehen lassen können.

Wie bereits 2010 ist die SÄZ auch 2017 die meistgelesene medizinische Fachzeitschrift der Schweiz: 94\% der Ärztinnen und Ärzte lesen sie zumindest gelegentlich oder blättern sie durch (Weitester Leserkreis WLK), und stolze
60\% können mit jeder Ausgabe erreicht werden (Reichweite). Diese Grössen müssen zwingend erhoben werden, damit eine Umfrage den Qualitätskriterien der WEMF [4] genügt, die in der Schweizer Medienforschung das Mass der Dinge ist. Sie sagen aber noch nichts darüber aus, welche Rubriken gelesen werden - möglicherweise sind dies ja nur die Stellenanzeigen ...

Dem ist nicht so. Gemäss der Umfrage lesen hochgerechnet beispielsweise 2300 Leserinnen und Leser jedes «Zu guter Letzt» - also diese Kolumne. Nimmt man noch die regelmässigen und gelegentlichen Leser dazu, sind es schon gegen 20000 . Wer nun glaubt, damit liege diese Rubrik an der Spitze des Leserinteresses, irrt. Freuen kann sich die FMH: Ihr Editorial und die standespolitischen Informationen erreichen gegen 5000 Stammleser und bis zu 29000 regelmässige und gelegentliche Leserinnen und Leser. Auf ähnlich gute Werte kommen die Standpunkte sowie Reportagen und Interviews im hinteren Teil der SÄZ. Besonders erfreulich: Keine Rubrik fällt total ab - selbst der «Kulturteil» erreicht über 1000 regelmässige und rund 16000 gelegentliche Leser. Mit diesem Nutzungsverhalten im Einklang steht, dass rund 80\% der Befragten mit dem thematischen Mix der SÄZ zufrieden sind. Hoch gewichtet die Leserschaft die Meinungsfreiheit: $85 \%$ finden es sehr wichtig, dass die SÄZ offen bleibt für freie Beiträge aller Art.

Erfreulich aus Sicht der Redaktion ist insbesondere, dass wir als standes- und gesundheitspolitische Zeitschrift auch die jungen Leserinnen und Lesern (<35 Jahre) erreichen. Dennoch haben wir uns vorgenommen, für diese Zielgruppe noch attraktiver zu werden, auch mit zusätzlichen Features im Internet und seit kurzem einem Facebook-Auftritt.

Fazit: Die SÄZ ist auf Kurs, doch wir wollen uns nicht auf eingebildeten Lorbeeren ausruhen. Ich wünsche Ihnen, liebe Leserin, lieber Leser, eine frohe Weihnachtszeit und einen guten Start ins neue Jahr.

\section{Literatur}

1 Kurt H. 20 Jahre EMH. Schweiz Ärztezeitung. 2017;98(51-52):17321734.

2 Scholer M. Von dunklen Wolken und blauem Himmel. Schweiz Ärztezeitung. 2017;98(50):1693-1695.

3 Eine Zusammenstellung der wichtigsten Ergebnisse der Befragung finden Sie online auf der SÄZ-Website unter www.saez.ch $\rightarrow$ Tour d'horizon $\rightarrow$ gfs-Leserumfrage.

4 WEMF steht für Werbemedienforschung. Aufgabe der WEMF AG ist es, systematisch, kontinuierlich und neutral zu erheben, wie Medien genutzt werden. 